\title{
Interaction between main line standing waves and side branch resonance frequencies
}

\author{
P. McKinnis, B. Miller \& A. Ruggles \\ University of Tennessee, Department of Nuclear Engineering, \\ Knoxville, USA
}

\begin{abstract}
Acoustic standing waves may be established in main gas delivery lines and are caused by resonance in branch lines. The branch line resonance frequency is normally determined from the geometry of the branch. Data are presented that show the branch line standing wave couples with the main line standing wave, and a range of side branch resonance frequencies are possible, with multiple frequencies existing in the side branch at the same time in some cases. This phenomenon is important to gas delivery systems where acoustic frequencies and loads must be predicted to facilitate design of the system components.

Keywords: branch line resonance, acoustics, standing waves, fluid-structure interaction.
\end{abstract}

\section{Introduction}

Small branch lines off larger main delivery lines can exhibit acoustic resonance. Branch lines with a reflective obstruction, such as a valve or instrument, are susceptible to a $1 / 4$ wavelength standing wave, with a pressure node positioned near where the branch meets the main line, and a pressure anti-node positioned at the obstruction. Of course, higher modes are possible with an odd number of quarter wavelengths existing in the branch such that,

$$
f_{\text {natural }}=\frac{n c}{4 L} \quad n=1,3,5,7 \ldots .
$$

where $c$ is the sound speed and the characteristic length, $L$, is the branch length. The branch length is sometimes extended by some fraction of the diameter. 
The most common drive for resonance in the branch line is vortex shedding at the branch, where vorticity in the fluid near the wall of the main line becomes free at the branch, and diverts flow periodically into the downstream branch wall, creating periodic pressure perturbations that serve as the energy source for the standing wave in the branch, Rockwell and Naudacher [1]. The frequency of pressure pulses created by the vortex shedding is predicted using the Strouhal number, $\mathrm{St}=\mathrm{fd} / \mathrm{v}$, with the branch diameter providing the length scale, and $\mathrm{v}$ set to the flow velocity in the main line. Coupling between the branch acoustic response and the vortex shedding behaviour allow for a branch to resonate for values for Strouhal number ranging roughly from 0.2 to 0.6 , Ziada and Shine [2]. Strouhal number near 0.4 normally gives the largest amplitude acoustic response in the branch. These values vary with system parameters such as Reynolds number, as examined by Ziada and Buhlmann [3] Bruggeman [4] and Kriesels et al [5].

Some previous applications have motivated enhancements to the basic theory to accommodate system specific attributes. The position of the branch downstream of a fitting such as a valve, orifice or elbow has been shown to influence vortex shedding and the associated Strouhal number response, Ziada and Shine [2], Lamoureux and Weaver [6]. Higher order vortex shedding modes are possible, leading to excitation of acoustic frequencies in branch lines at higher Strouhal numbers, Ziada [7]. Branches positioned across from each other or in tandem may interact to complicate and enhance the acoustic response of the system, Ziada and Buhlmann [3]. Modifications to the geometry of the branch junction to the main line have been explored to mitigate vortex shedding as a coherent source of energy for driving branch line resonance, Weaver and MacLeod [8].

While the basic theory provides design guidance associated with the resonance in the branch, the excitation of standing waves in the main line has received little attention. A few studies examine acoustic coupling of branch line modes through the main line, Coffman and Bernstein [9], Ziada and Buhlmann [3], and Ziada and Shine [2]. However, these studies remain concerned with the loading of components in the branch line, such as safety relief valves. Additional knowledge of acoustic loads in the main line is desired for capital intensive applications where more flexibility in design and operation is desired, such as steam supply systems. Components in the steam supply, such as super-heater bundles, steam separator assemblies and steam dryer assemblies may have many structural resonances. Acoustic drives near structural resonance may lead to fatigue failures and commercial performance shortfalls due to loss of equipment availability and reduced component endurance. Avoidance of acoustic excitations corresponding to all or specific structural resonances for conditions of sustained operation is desired. A comprehensive engineering formalism for identifying acoustic oscillations caused by side branches that integrate to cause significant acoustic pressure levels in the main line would be useful for this purpose.

Past efforts have focused on the energy budget of the branch line only, with the vortex shedding mechanism as the energy source, and the energy sinks 
including damping mechanisms in the branch and acoustic radiation into the main line, Bruggeman [4] and Kriesels et al [5]. In circumstances where damping is low, and outlet paths for the acoustic energy in the main line are limited, the amplitude of the standing wave pattern in the main line can grow quite large, especially if multiple branches are present. Pressure variations in the main line at the branch position influence the acoustic performance of the branch and can modify the branch resonant frequency. This paper explores adjustments to the basic theory to correct for the influence of a standing wave in the main line. The relationship between the standing wave pattern in the main line and the side branch resonance is evaluated through experiment and one dimensional linear acoustic theory. The motivation for the investigation and development is design of nuclear steam supplies, but the modelling outcomes have more general applicability.

The development begins by examining how the main line pressure field may influence the resonant frequency of the branch. The standing wave in the main line is then simulated in the presence of Doppler shift, which modulates the standing wave amplitude with position in the main line. Finally, a low pressure air experiment is presented that provides a simple, easily modified environment to examine the theory.

\section{Wave forms in main lines and how they interface with branch positions}

Standing waves of one-quarter wavelength may be induced in closed branch lines as represented in equation (1) for the primary mode with $n$ equal unity. The model assumes that the ratio of the diameter of the branch line over the diameter of the main line is small, such that the pressure at the branch line entrance is near zero. In the case where standing pressure waves build in the main line, the pressure at the inlet of the branch line is equal to the pressure in the main line at the location of the branch. Figure 1 shows the relationship between pressure oscillations in the main line on the wavelength of the standing wave in the branch line.

From Figure 1, the length of the branch line equal to one-quarter of the primary mode wavelength holds true when the branch line is located over a pressure node in the main line. However, as the acoustic pressure in the main line moves towards an antinode, the wavelength of the standing wave in the branch either increases - moving out into the main line and remaining in phase with the pressure variation in the main line, or decreases - moving into the branch line and operating out of phase with the main line pressure response.

Because of this phenomenon, the natural frequency of the branch line is expected to shift depending on its location relative to the standing wave pattern in the main line. If a term, $\beta$, is defined as,

$$
\beta=L-\frac{1}{4} \lambda
$$


then standing waves are induced in the branch line when the natural frequency corresponds to the length of the branch line in the following way:

$$
f_{\text {natural }}=\frac{n c}{4(L-\beta)} \quad n=1,3,5,7 \ldots . .
$$

\section{Lengthening $(L<0.25 \lambda) \quad$ Textbook $(L=0.25 \lambda) \quad$ Shortening $(L>0.25 \lambda)$}

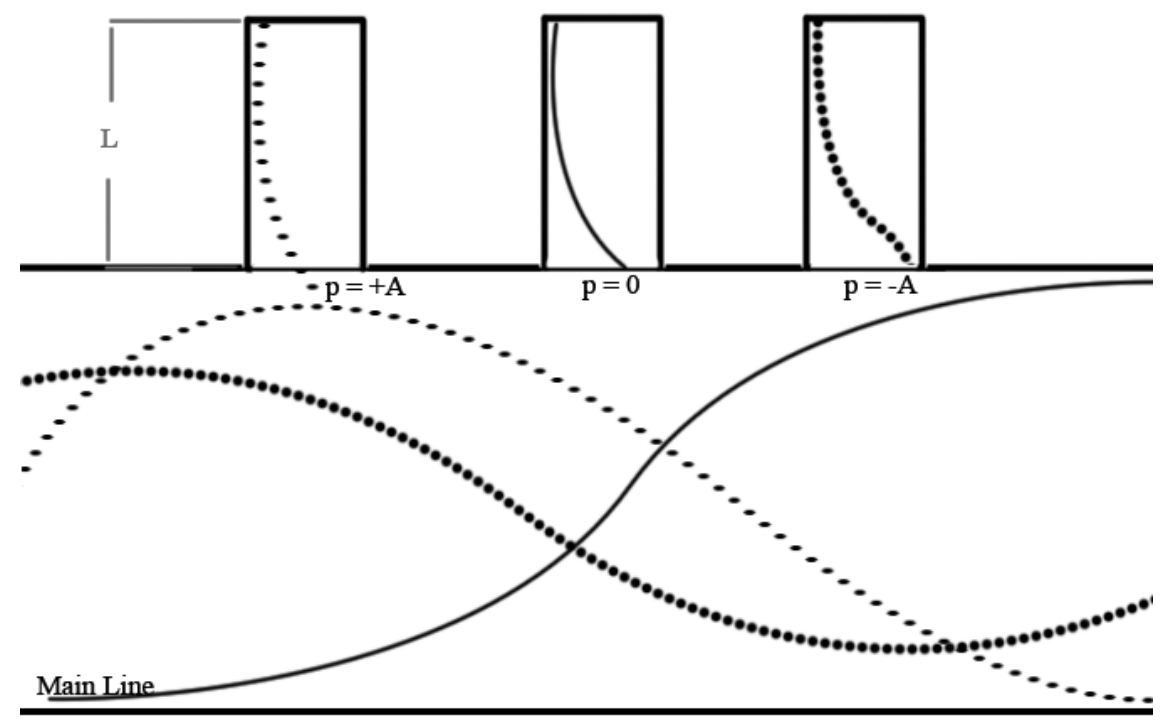

Figure 1: Lengthening and shortening of standing waves when subjected to pressure oscillations in the main line.

This is a manipulation of equation (1). For the fundamental mode, the value of $\beta$ can be determined from the pressures at the inlet and top of the branch line using the following:

$$
\beta=\frac{2 \theta L}{2 \theta+\pi}
$$

where $\theta$ is defined as,

$$
\theta=\sin ^{-1}\left(\frac{P_{\text {inlet }}}{P_{\text {max }}}\right)
$$

$P_{\text {inlet }}$ is the pressure at the inlet of the branch line and $P_{\max }$ is the pressure at the top of the branch line. 


\section{Examination of the Doppler effect in the main line}

The branch line is not stationary with respect to the steam flow. Therefore, the pressure wave caused by the branch in the main line is shifted in wavelength because of the Doppler effect. Upstream of the branch line, the wavelength is shorter, and downstream of the branch line, the wavelength is longer. Note that in this application, from the perspective of a stationary observer (i.e., instrumentation, branch position or Eulerian reference), the wavelength is shifted, not the frequency. The Doppler shift in wavelength can be written as a function of Mach number:

$$
\begin{aligned}
& \lambda_{d s}=\lambda(1+M) \\
& \lambda_{u s}=\lambda(1-M)
\end{aligned}
$$

where $\lambda_{d s}$ is the shifted wavelength downstream of the branch line, $\lambda_{u s}$ is the Doppler shifted wavelength upstream from the branch line, $\lambda$ is the unshifted wavelength, and $M$ is the Mach number. Thus the normalized pressure wave can be written as a simple sine function.

$$
\begin{aligned}
& P_{u s}=\sin \left(\frac{2 \pi x}{\lambda_{u s}}\right) \\
& P_{d s}=\sin \left(\frac{2 \pi x}{\lambda_{d s}}\right),
\end{aligned}
$$

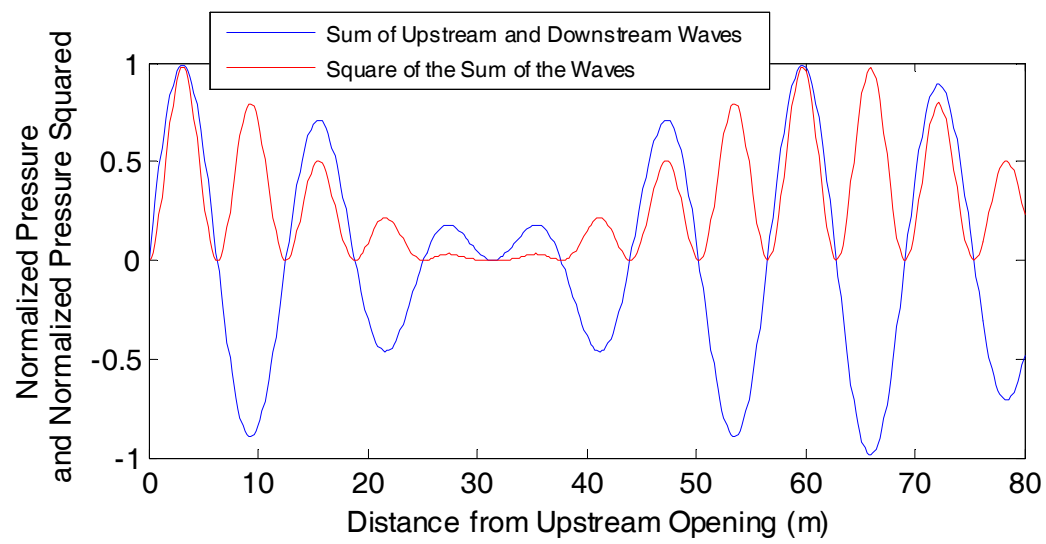

Figure 2: Interaction of upstream and downstream Doppler shifted waveforms.

The sum of the upstream and downstream travelling waves causes a composite wave form inside of the main line. An example of this type of waveform is shown in Figure 2 with the left boundary corresponding to a 
compliant interface. If the branch were positioned near $60 \mathrm{~m}$ for the example system in Figure 2, then one would expect some shift in branch resonance frequency, or perhaps two distinct frequencies may be observed in the branch as discussed in section 2 of this paper.

\section{Experimental}

The application of interest in this effort is a steam supply with several branch lines and other flow management hardware such as valves and fittings. Early examination of acoustic pressures at discrete locations on the main steam lines exposed very complex behaviour. A simple, atmospheric pressure, air test facility was developed to examine basic attributes of the relationship between standing waves in the main line and the branch line.

\subsection{Experimental facility}

The experimental setup shown in Figure 3 consists of a main line $297 \mathrm{~cm}$ long made of sections of $7.6 \mathrm{~cm}$ ( 3 in) diameter PVC piping connected to a blower via flexible aluminium tubing. Air was drawn through the apparatus from section 1 as labelled in Figure 3, toward the Dayton ${ }^{\mathrm{TM}}$ model 3N669A $3.7 \mathrm{~kW}$ blower, section 12. Velocities up to $43 \mathrm{~m} / \mathrm{s}$ are possible. Sections $1,3,6$, and 8 are 7.6 $\mathrm{cm}$ diameter Schedule 40 PVC piping. These sections were interchangeable so as to vary the relative position of the branch line with respect to the upstream and downstream boundary. The upstream boundary, positioned left of section 1, was open to the room and contained no obstructions for several diameters in every direction. The downstream boundary was a transition from PVC piping (section 10) to flexible corrugated aluminium tubing (section 11). Sections 2,4,7, and 10 represent couplings. Section 5 represents a short capped branch line made of $1.9 \mathrm{~cm}(3 / 4$ in) PVC pipe. The branch was carefully prepared by drilling a hole through the pipe and solvent welding the branch on top of the hole. This yielded a sharp branch edge as shown in Figure 4.

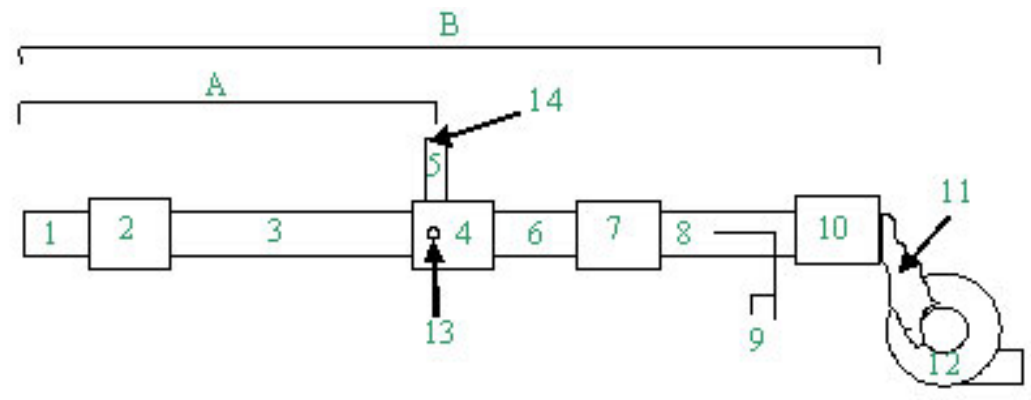

Figure 3: Diagram of experimental apparatus. 


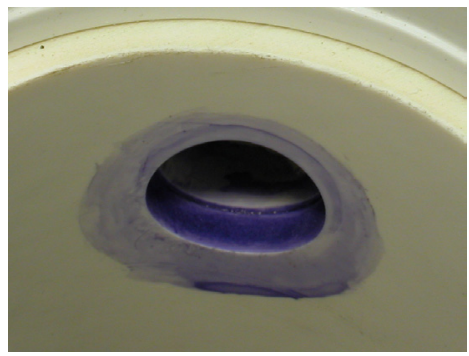

Figure 4: A view from the inside of the pipe of the sharp branch edge.

The apparatus contains 2 through wall mounted PCB Piezotronics ${ }^{\mathrm{TM}}$ model 130D20 microphones, located at positions 13 and 14 in Figure 3, and another identical microphone on a measuring tape that could be moved in the main line. A pitot tube was used to measure fluid velocity and is shown at position 9 . It was connected to an Omega ${ }^{\mathrm{TM}}$ model PX653-05D5V electrical differential pressure cell that had a response time of $250 \mathrm{~ms}$. The microphone was positioned so that the diaphragm was located in the stream just below the branch line. It was sampled at $100 \mathrm{kS} / \mathrm{s}$ with a National Instruments DAQ model NI USB-9162.

\subsection{Matrix for data acquisition}

The behaviour of the system can be complex, and it is desired to characterize the system performance thoroughly. Two initial permutations of the facility geometry were planned and are reported here, both conducted at a constant main line flow velocity of $38 \mathrm{~m} / \mathrm{s}$, corresponding to a flow Mach number of 0.112 .

\subsubsection{Experiment 1 - constant length}

The position of the branch line, section 5, was varied by changing the length of pipes 1 and 6 . They were changed such that the total length from the front edge of pipe 1 to the back edge of coupling 10 (Length B) remained constant at $297 \mathrm{~cm}$. Ten experiments were done such that the distance from the upstream boundary to the centre of the branch line was varied from $199 \mathrm{~cm}$ to $222 \mathrm{~cm}$ in $2.54 \mathrm{~cm}$ increments. For each setup, the stream velocity and pressure were measured in two-second packets. The pressure was sampled at $100 \mathrm{kS} / \mathrm{s}$. Twenty such data packets were measured and analyzed for each experimental setup.

\subsubsection{Experiment 2 - variable length}

The position of the branch line was varied relative to the upstream boundary by changing the length of pipe 1 (Length A). However, the position of the branch relative to the downstream boundary remained constant, causing a change in the total length of the pipe (Length B). Once again, ten pipe configurations were explored. In each successive setup, the length of pipe 1 was lengthened by $2.54 \mathrm{~cm}$, causing the distance from the upstream boundary to vary from 199 to 
$222 \mathrm{~cm}$. The distance from the pipe to the downstream boundary was held constant at $58.4 \mathrm{~cm}$. Once again twenty, two-second, data packets were obtained for each piping configuration and the pressure was sampled at $100 \mathrm{kS} / \mathrm{s}$.

\subsection{Data reduction and calculations}

The branch line used for reported data was $16.0 \mathrm{~cm}$ long. Therefore, with sound speed equal to $340 \mathrm{~m} / \mathrm{s}$ the expected frequency is $531 \mathrm{~Hz}$. The pressure data is processed in two-second time series packets. A Hanning window is applied to each packet and a Fourier transform performed using Matlab ${ }^{\mathrm{TM}}$. The peaks near $531 \mathrm{~Hz}$ were examined manually.

\subsection{Results}

The time series pressure data from the microphone positions are treated in the frequency domain. Two second time series are used throughout this evaluation, but other time series lengths were examined before selecting this time window as an appropriate and representative value.

\subsubsection{Experiment 1}

In experiment 1, the waveform produced by the branch line had a great deal of variance. Six of the ten configurations showed a single frequency peak near the expected value. Figure 5 shows the power density spectra typical of all six of these configurations. The frequency varied slightly in these experiments from 511 to $516 \mathrm{~Hz}$.

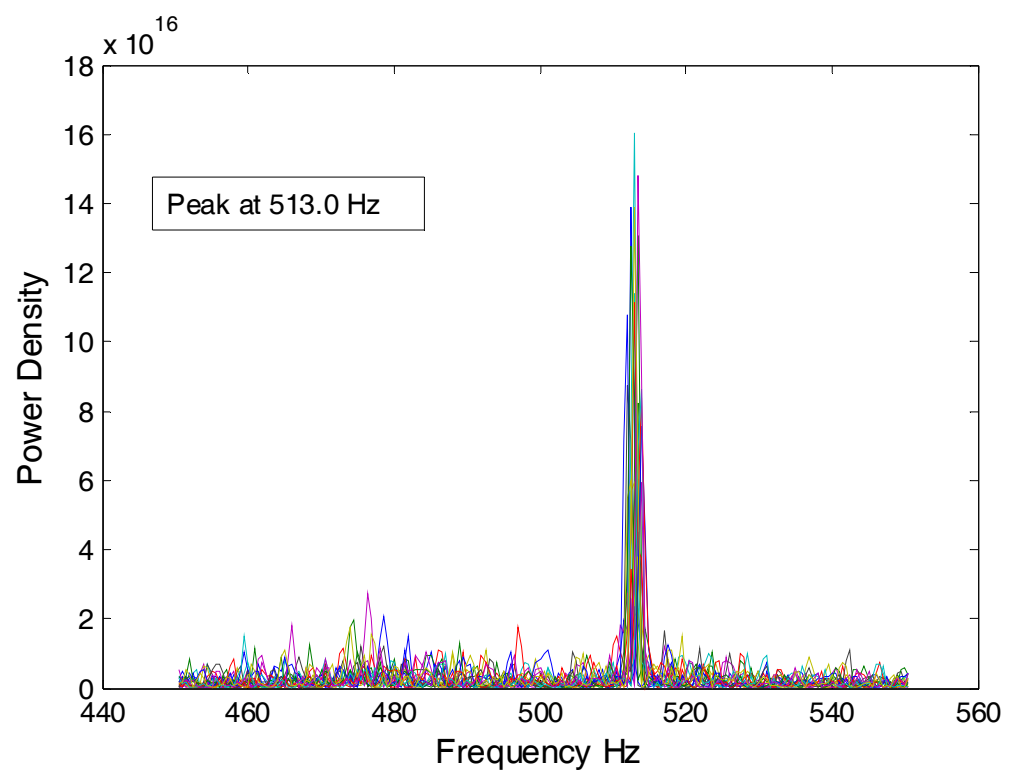

Figure 5: $\quad$ Typical single peaked power spectra with peak near the expected value - all 20 data packets graphed individually. 


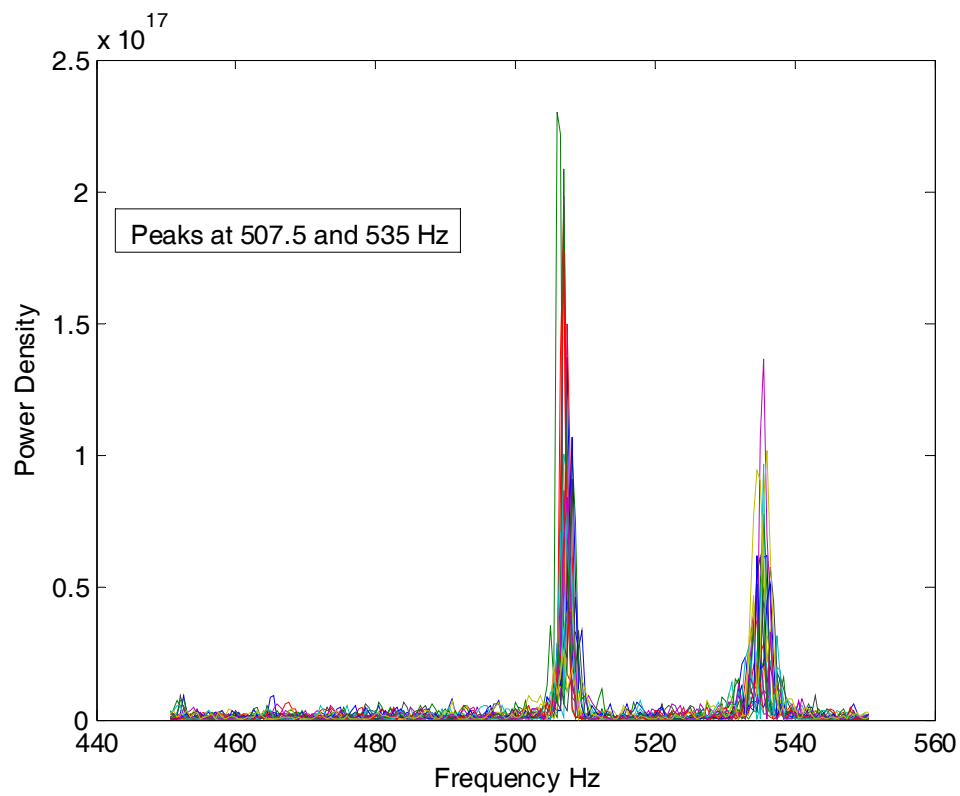

Figure 6: Typical 2 peaked power spectra - 20 data packets graphed individually.
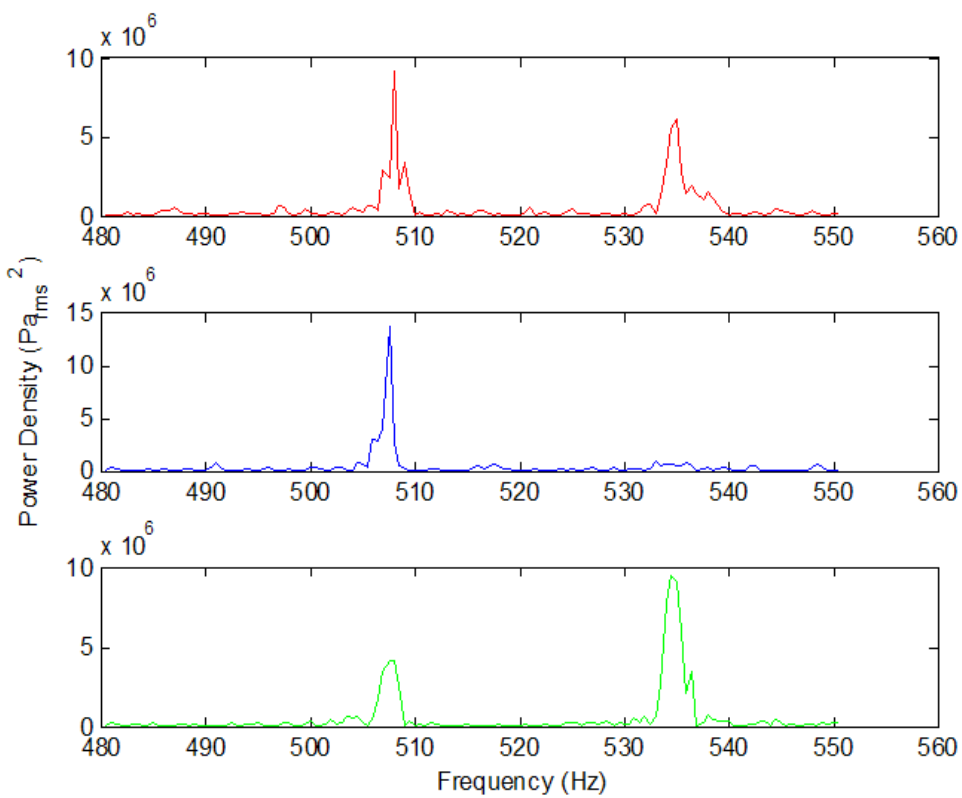

Figure 7: Shifting of branch energy between multiple peaks in time: (a) (top) peaks are typical of time averaged values; (b) (middle) only the low frequency peak is expressed; (c) (bottom) the high frequency peak has a greater amplitude. 
Four of the ten configurations showed two significant frequency peaks. Figure 6 shows spectra typical of these configurations. From Figure 6, it may seem that all twenty of the data packets for the given configuration express both peaks. However, some do not express the smaller high frequency peak as can be seen in Figure 7(b). Additionally, some data packets express a low frequency peak that is smaller than the high frequency peak, as shown in Figure 7(c). The waveform shifts in expression between the low and high frequency peak over relatively long time periods.

\subsubsection{Experiment 2}

In this experiment, with the position of the branch relative to the downstream end of the main line fixed, all configurations had stable, high-amplitude peaks, with Figure 8 showing typical power spectra. As the branch line position was varied, the branch frequency, and the frequency in the main line, varied over $5.5 \%$. This variance is a near-linear function of the main line length as shown in Figure 9. Note that between $215 \mathrm{~cm}$ and $217 \mathrm{~cm}$ the frequency promptly increases before beginning to decrease again.

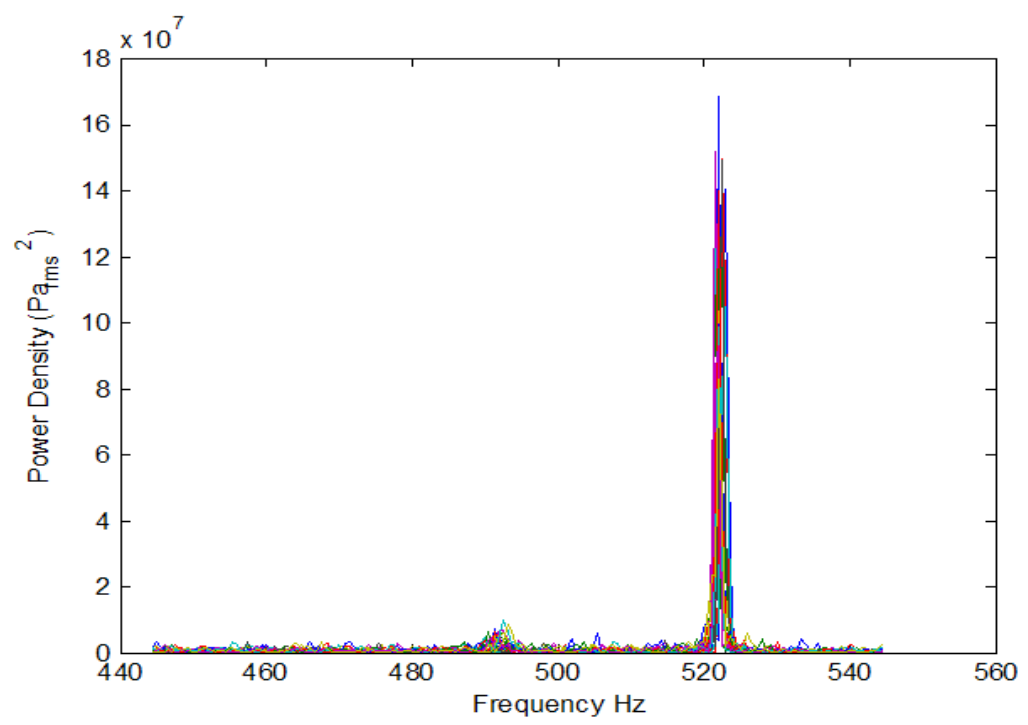

Figure 8: Branch power density with branch $215 \mathrm{~cm}$ from upstream boundary -20 data packets graphed individually.

The frequency content of the pressure at the end of the branch closely corresponds to the behaviour of the pressure at the base of the branch for all the geometries evaluated. 


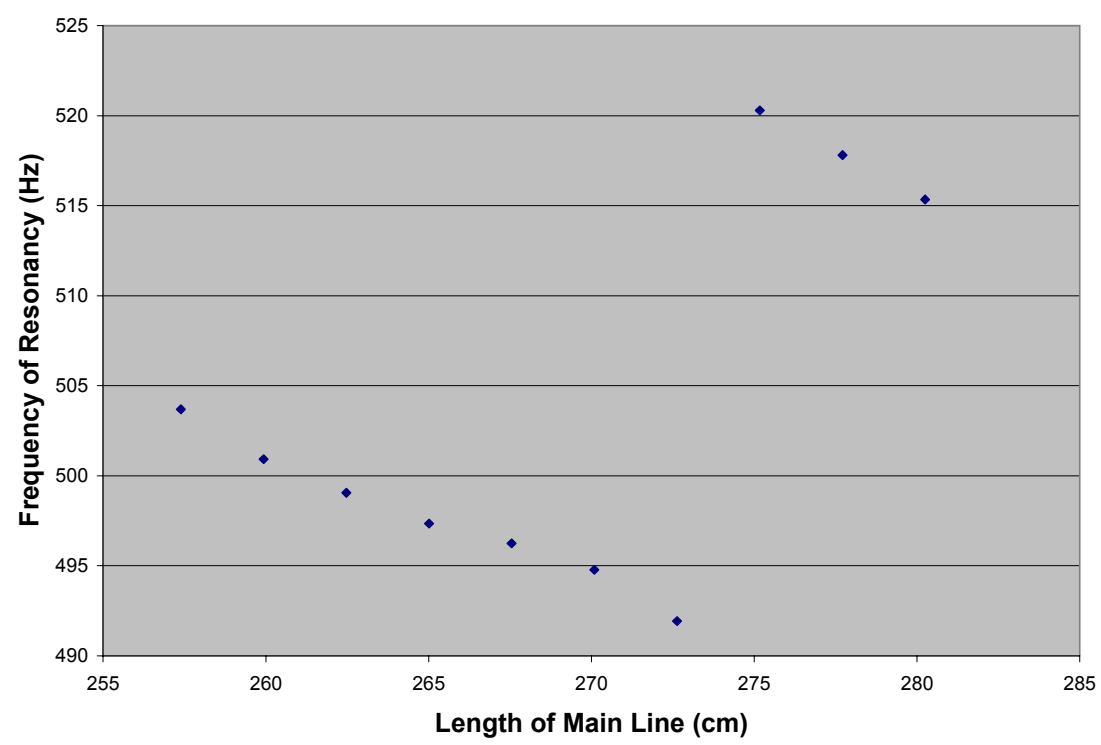

Figure 9: Frequency shift due to variation of main pipe length.

\section{Interpretation of results}

The main line has compliant boundary conditions at each end, and therefore has resonant frequencies corresponding to situations where an integer number of half wavelengths exist in the line,

$$
f=\frac{c n}{2 L_{m}} \quad n=1,2,3, \ldots
$$

The drive frequency from the branch supplies energy to the resonant frequency in the main line that closely matches the branch resonance frequency. Variation in the main line length varies the main line resonance frequency, as shown clearly in Figure 9. The frequency jumps to change the number of half wavelengths in the main line when the main line resonance departs too far from the preferred branch drive frequency. The transition depicted in Figure 9 near main line length of $274 \mathrm{~cm}$ corresponds to a transition from $n$ equal nine to $n$ equal ten in equation (10). The branch drive frequency and the main line resonance lock-in, with both the branch reflective end pressure, and main line pressure exhibiting the same primary frequency content. The wavelength in the branch line and the wavelength in the main line move from their preferred independent values to create a single resonance frequency for the system.

In the first experiment, where the branch position is varied in a main line of fixed length, there are branch locations where two primary resonance frequencies exist in the main line. The branch appears to be conflicted about which 
frequency to prefer, wandering relatively slowly between two distinct values. More investigation is planned to better characterize and understand this behaviour.

\section{Conclusions}

Side branch resonance frequencies couple with main line resonance frequencies, allowing a branch to resonate over a range of frequencies as the length of the main line is altered. This is important to engineering applications where the resonant frequency of a branch must be predicted with some accuracy. Current engineering practice does not include consideration of the standing wave patterns in the main line when branch line resonance is evaluated. This can lead to significant errors when conditions are favourable for a standing wave pattern to build in the main line.

The acoustic energy balance for the branch and main line together includes a source term associated with vortex shedding at the branch, and damping mechanisms and acoustic radiation from the main line ends. Wave amplitudes will build in the branch and main line when damping and radiation terms are small relative to the source terms, until a steady state energy balance is achieved. The steady state pressure amplitudes tend to increase as the system pressure or gas density is increased due to the behaviour of the source term relative to the damping and radiation terms, Buggeman [4] and Kriesels et al [5]. This implies the frequency coupling observed in the low pressure air facility may be more pronounced in higher pressure situations.

The pressure amplitudes in the main line of this experiment are too low relative to the branch line pressures to allow examination of branch frequency shifting that may occur due to pressure variation in the main line, as proposed in section 2 of this paper. However, significant wavelength and associated frequency accommodation appears to be possible in the branch line and main line to allow lock-in of a single, system-wide, resonance frequency. The branch line resonance frequency is coupled to the resonance frequency of the main line, and follows the resonance frequency of the main line as the main line length is varied. The branch resonance frequency varied over a six percent range in this experiment. However, larger ranges are likely for other configurations of branch line length and main line length. The coupling may also be more pronounced in high pressure systems, allowing branch resonance frequencies to vary over larger spans. This has important implications when resonant frequencies are being evaluated for use in predicting structural loadings in design of large systems.

\section{References}

[1] Rockwell, D. and Naudascher, E., "Review-Self Sustaining Oscillations of Flow Past Cavities." Journal of Fluids Engineering, Vol. 100, 152-165, 1978. 
[2] Ziada, S. and Shine, S., "Strouhal Numbers of Flow-Excited Acoustic Resonance of Closed Side Branches." Journal of Fluids and Structures, Vol. 13, 127-142, 1999.

[3] Ziada, S. and Buhlmann, E. T., "Multiple Side-Branches as Tone Generators." Proceedings of International Mechanical Engineering Conference, paper 0416/009, pp. 435-444, 1991.

[4] Bruggeman, J. C., "Flow Induced Pulsations in Pipe Systems." PhD Thesis, Technische Universiteit Eindhoven, 1987.

[5] Kriesels, P. C., Peters, M. C. A. M., Hirschberg, A., Wijnands, A. P. J., Iafrati, A., Riccardi, G., Piva, R., Bruggeman, J. C., "High Amplitude Vortex Induced Pulsations in Gas Transport Systems." Journal of Sound and Vibration, Vol. 184, 343-368, 1995.

[6] Lamoureux, P. N. and Weaver, D. S., "The Effects of Turbulence and Damping on Pipeline Acoustic Resonance." Proceedings of the Institution of Mechanical Engineers, Flow Induced Vibrations, paper C416/093, May 20-22, 1991.

[7] Ziada, S., "A Flow Visualization Study of Flow-Acoustic Coupling at the mouth of a Resonant Side Branch." Journal of Fluids and Structures, Vol. 8, 391-416, 1994.

[8] Weaver, D. S., and MacLeod, G. O., "Entrance Port Rounding Effects on Acoustic Resonance in Safety Relief Valves." Pressure Vessels and Piping, Vol 389, Flow-Induced Vibration, 291-296, 1999.

[9] Coffman, J. T. and Bernstein, M. D., "Failure of Safety Valves Due to Flow Induced Vibration." Journal of Pressure Vessel Technology, Vol. 102, 112118,1980 . 